\title{
Force Field Comparison of GM1 in a DOPC Bilayer Validated With AFM and FRET Experiments
}

Michael C. Owen*1,4, Andreas Karner², Radek Šachl ${ }^{3}$, Johannes Preiner ${ }^{2}$, Mariana Amaro ${ }^{3}$, Robert Vácha ${ }^{1,4}$

${ }^{1}$ CEITEC - Central European Institute of Technology, Kamenice 5, 62500 Brno, Czech Republic

${ }^{2}$ University of Applied Sciences Upper Austria, 4020 Linz, Austria

${ }^{3}$ Department of Biophysical Chemistry, J. Heyrovský Institute of Physical Chemistry of the C.A.S., v.v.i., Dolejškova 2155/3, Prague, Czech Republic

${ }^{4}$ Faculty of Science, Masaryk University, Kamenice 5, 62500 Brno, Czech Republic

* To whom correspondence should be addressed.

\section{Email: michaelowen27@gmail.com}

SUPPLEMENTARY INFORMATION 


\section{METHODS}

The distance between restrained groups in umbrella sampling simulations

The distances (in $\mathrm{nm}$ ) between the center of mass of the restrained groups AC3 or GAL5 and DOPC for the umbrella sampling simulations using each force field are as follows:

\section{CHARMM:}

AC3 and DOPC: 1.47, 1.54, 1.64, 1.71, 1.84, 1.88, 2.00, 2.01, 2.10, 2.13, 2.14, 2.24, 2.29, 2.32, 2.39, 2.46, 2.50, 2.55, 2.58, 2.59, 2.71, 2.74, 2.75, 2.87, 2.88, 2.94, 2.95, 3.26, 3.27, 3.43, 3.44, 3.54, 3.62. GAL5 and DOPC: 1.99, 2.02, 2.15, 2.22, 2.28, 2.32, 2.36, 2.39, 2.49, 2.52, 2.58, 2.63, 2.64, 2.72, 2.74, 2.79, 2.87, 2.94, 2.96, 2.98, 3.06, 3.11, 3.17, 3.25, 3.31, 3.34, 3.44, 3.46, 3.56, 3.59, 3.64, 3.76.

\section{GROMOS:}

AC3 and DOPC: 1.07, 1.09, 1.12, 1.13, 1.19, 1.22, 1.25, 1.30, 1.33, 1.37, 1.43, 1.46, 1.52, 1.59, 1.63, 1.69, 1.75, 1.83, 1.89, 1.91, 1.91, 1.96, 2.04, 2.09, 2.11, 2.12, 2.28, 2.30, 2.32, 2.38, 2.45, 2.47, 2.58, 2.61, 2.65, 2.73, 2.76, 2.81, 2.88, 2.96, 3.00, 3.06, 3.10, 3.20, 3.23, 3.32, 3.36, 3.42, 3.50, 3.55.

GAL5 and DOPC: 1.06, 1.09, 1.10, 1.13, 1.18, 1.22, 1.26, 1.28, 1.31, 1.34, 1.41, 1.45, 1.49, 1.53, 1.57, $1.61,1.72,1.73,1.80,1.82,1.90,1.92,1.95,2.08,2.12$, 2.19, 2.20, 2.23, 2.28, 2.31, 2.33, 2.39, 2.45, 2.50, 2.51, 2.60, 2.61, 2.67, 2.77, 2.83.

\section{LIPID14:}

AC3 and DOPC: 1.89, 1.90, 1.98, 2.04, 2.06, 2.09, 2.10, 2.20, 2.23, 2.24, 2.27, 2.33, 2.34, 2.37, 2.42, 2.49, 2.50, 2.51, 2.56, 2.62, 2.66, 2.73, 2.76, 2.79, 2.85, 2.89, 2.93, 2.95, 2.97, 2.98, 3.04, 3.09, 3.11, 3.15, 3.20, 3.21, 3.28, 3.29, 3.34, 3.40, 3.43, 3.47, 3.51, 3.52, 3.55, 3.60, 3.66.

GAL5 and DOPC: 1.66, 1.69, 1.72, 1.78, 1.84, 1.88, 1.92, 1.96, 2.00, 2.04, 2.10, 2.11, 2.14, 2.21, 2.26, 2.30, 2.36, 2.41, 2.47, 2.52, 2.57, 2.60, 2.64, 2.70, 2.74, 2.78, 2.80, 2.86, 2.90, 2.93, 2.97, 3.00, 3.05, 3.13, 3.16, 3.21, 3.29, 3.34, 3.39, 3.44, 3.48, 3.55, 3.62, 3.66, 3.74, 3.79, 3.86 .

\section{OPLS:}

AC3 and DOPC: 1.44, 1.50, 1.54, 1.59, 1.70, 1.75, 1.76, 1.80, 1.89, 1.99, 2.03, 2.05, 2.09, 2.11, 2.19, 2.21, 2.22, 2.37, 2.42, 2.51, 2.54, 2.55, 2.70, 2.73, 2.77, 2.86, 2.90, 2.91, 2.98, 3.04, 3.07, 3.10, 3.17, 3.20, 3.27, 3.29, 3.32, 3.36, 3.40, 3.49, 3.55 .

GAL5 and DOPC: 1.23, 1.31, 1.37, 1.44, 1.46, 1.58, 1.60, 1.62, 1.65, 1.67, 1.79, 1.81, 1.92, 1.94, 2.00, 2.04, 2.13, 2.15, 2.17, 2.23, 2.27, 2.38, 2.42, 2.55, 2.59, 2.67, 2.69, 2.82, 2.90, 2.99, 3.07, 3.09, 3.15, 3.20, 3.22, 3.41, 3.49, 3.55, 3.70.

\section{SLIPIDS:}

AC3 and DOPC: 1.12, 1.25, 1.32, 1.35, 1.38, 1.41, 1.47, 1.50, 1.57, 1.60, 1.64, 1.69, 1.73, 1.79, 1.80, $1.82,1.87,1.92,1.93,1.95,1.98,2.03,2.09,2.15,2.21,2.25,2.31,2.35,2.44,2.50,2.54,2.59,2.64$, 2.70, 2.76, 2.83, 2.91, 2.94, 2.98, 3.02, 3.08, 3.15, 3.20, 3.26, 3.34, 3.43, 3.59, 3.66.

GAL5 and DOPC: 1.30, 1.32, 1.36, 1.47, 1.51, 1.54, 1.58, 1.64, 1.69, 1.74, 1.78, 1.80, 1.84, 1.87, 1.91, 1.98, 2.01, 2.04, 2.09, 2.16, 2.19, 2.24, 2.28, 2.31, 2.36, 2.44, 2.48, 2.51, 2.58, 2.61, 2.68, 2.70, 2.75, 2.83, 2.90, 2.94, 3.00, 3.03, 3.09, 3.12, 3.15, 3.22, 3.27, 3.32. 
Determining the distance of the GM1 headgroups from the lipid-water interface by FRET

Baumann and Fayer ${ }^{64}$ derived analytical equations that account for Förster resonance energy transfer (FRET) occurring between randomly distributed donors and acceptors within a plane (termed intra-FRET), but also between two different parallel planes (termed inter-FRET, Figure S1). By assuming dynamic limit conditions, where movements of dipoles are much faster than an energy transfer event, the so-called survival probability function for intra-FRET $G_{\text {intra }}$ (expressing the probability that an excited donor is still in the excited state) can be calculated:

$$
\ln G_{\text {intra }}(t)=-C_{2} \Gamma\left(\frac{2}{3}\right)\left(\frac{t}{\tau}\right)^{1 / 3}
$$

Here, $C_{2}$ is the reduced surface concentration of acceptors, which represents the average number of acceptors within the area of $\pi R_{0}^{2}, \Gamma$ is the gamma function and $\tau$ the average lifetime of donors in the absence of acceptors. Similarly, the survival probability for inter-FRET, $G_{\text {inter }}$, is given by:

$$
\ln G_{\text {inter }}(t)=\frac{-C_{2}}{3}\left(\frac{d_{x}}{R_{o}}\right)^{2}\left(\frac{2 \mu}{3}\right)^{1 / 3} \int_{0}^{2 / 3 \mu}\left(1-e^{-s}\right) s^{-4 / 3} d s
$$

where $d_{x}$ is the transversal distance between the layer of donors and the layer of acceptors, $\theta_{r}$ is the angle between the bilayer normal and the vector connecting the locations of the donor and acceptor dipoles, $\mu=3 t\left(\frac{R_{0}}{d}\right)^{6} \frac{1}{2 \tau}$ and $s=2 \mu \cos ^{6} \frac{\theta_{r}}{3}{ }^{64}$ Both inter- and intra-FRET can occur simultaneously (Figure S1). Then, the total survival probability is given by the joint probability $G(t)=G_{\text {intra }}(t) G_{\text {inter }}(t)$. The fluorescence intensity $F(t)$ of the donors in the presence of the acceptors is described as

$$
F(t)=G(t) \sum \alpha_{i} \exp \left(\frac{-t}{\tau_{i}}\right)
$$

where $\sum a_{i} \exp \left(-t / \tau_{i}\right)$ represents the decay of the donors in the absence of FRET.

To localize the GM1 headgroups in respect to the lipid-water interface, GM1 labelled in the headgroup by FL-Bodipy (g-GM1) was used as the donor and DiD as the acceptor. In this work, an assumption was made that the chromophores of DiD were localized at the lipid-water interface whereas the chromophores of g-GM1 were localized in two parallel layers at the distances $d_{\mathrm{A}}$ and $d_{\mathrm{B}}$ (Figure 
S2). Then, FRET occurs between a layer of donors and two other layers of acceptors, and leads to the following expression for $F(t)$ :

$$
F(t)=G_{\text {trans }}\left(d_{A}\right) G_{\text {trans }}\left(d_{B}\right) \sum \alpha_{i} \exp \left(\frac{-t}{\tau_{i}}\right) .
$$

$d_{\mathrm{A}}$ and $d_{\mathrm{B}}$ are related to the thickness of the lipid bilayer $d$ via $d_{\mathrm{B}}=d_{\mathrm{A}}+d$ (Figure S2). In this work, the GM1 head groups were localized by fitting calculated decays described by equation (4) to experimentally obtained decays. In this way, the parameters $d_{\mathrm{A}}, d$ and $C_{2}$ could be determined.

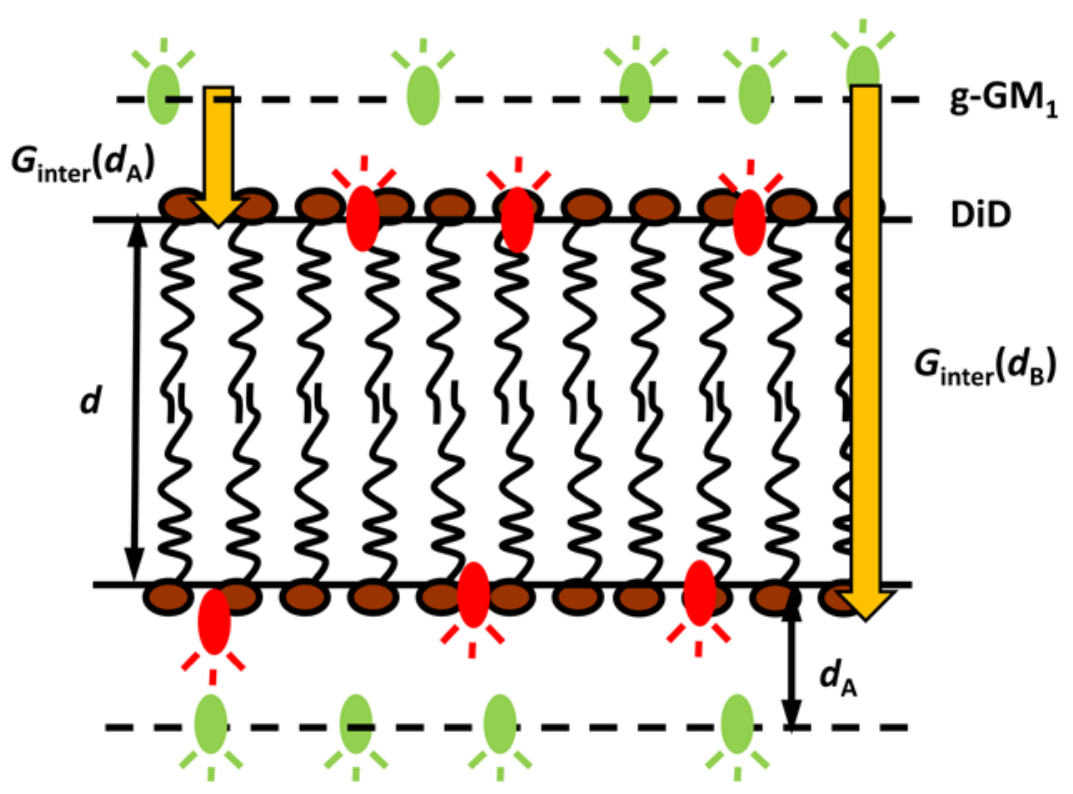

Figure S1: When donors (green ellipsoids) and acceptors (red ellipsoids) are localized in two parallel planes separated by a distance $d$, FRET occurs both within the same leaflet (intra-FRET) and between the two leaflets (inter-FRET). 


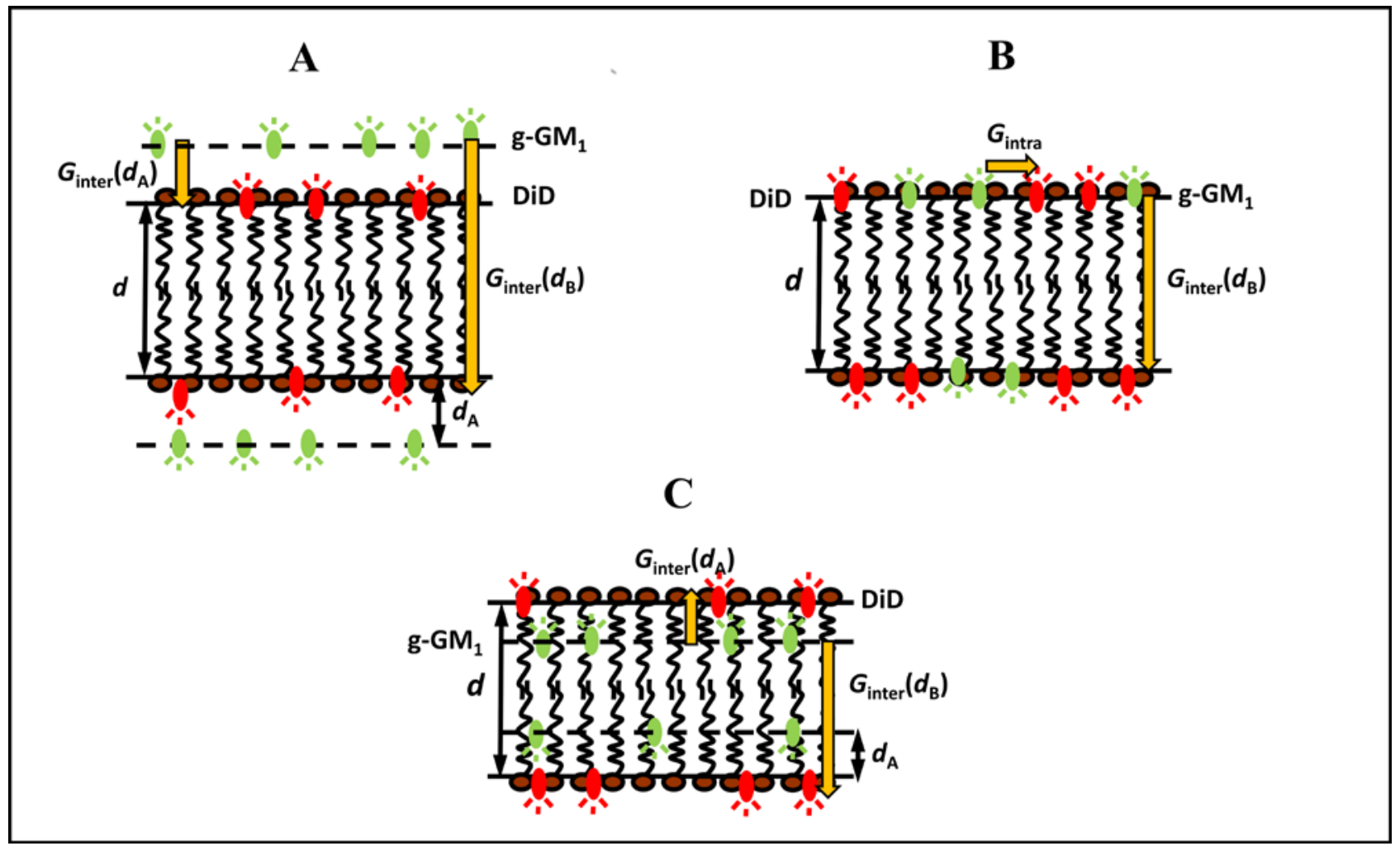

Figure S2. Experimental design of the FRET experiment. Donors were localized in two parallel planes whereas acceptors were localized in two other planes close to the lipid-water interface. FRET (characterized by the survival probabilities $G_{\text {inter }}\left(d_{\mathrm{A}}\right)$ and $G_{\text {inter }}\left(d_{\mathrm{B}}\right)$ ) occurs between a layer of donors and two other layers of acceptors. 


\section{RESULTS}

Supporting results of the FRET analysis

The global fits of the experimental time-resolved fluorescence decays of FL-Bodipy are shown in Figure S3. The output parameters of the fit were the transversal distance between the two DiD layers $d$ (which defines the thickness of the bilayer), the distance between the layer containing DiD and the closer layer containing g-GM1 $d_{\mathrm{A}}$, and the surface concentration of acceptors $C_{2}$. The values of these parameters are summarized in Table S1.

Table S1: Summary of the output parameters obtained from the global fit. Note that DiD was mixed with DOPC so as to roughly achieve the expected probe to lipid ratio. Therefore, the agreement between $C_{2}$ (expected) and $C_{2}$ (determined) is not expected to be perfect.

\begin{tabular}{ccccc}
\hline $\begin{array}{c}\text { Expected } \\
\text { probe to lipid } \\
\text { ratio }\end{array}$ & $\boldsymbol{C}_{2}$ (expected) & $\boldsymbol{C}_{2}$ (determined) & $\boldsymbol{d}_{\mathrm{A}} / \mathbf{n m}$ & $\boldsymbol{d} / \mathbf{n m}$ \\
\hline $1: 200$ & 0.58 & 0.81 & & \\
\hline $1: 300$ & 0.39 & 0.58 & & \\
\hline $1: 400$ & 0.29 & 0.42 & & \\
\hline
\end{tabular}

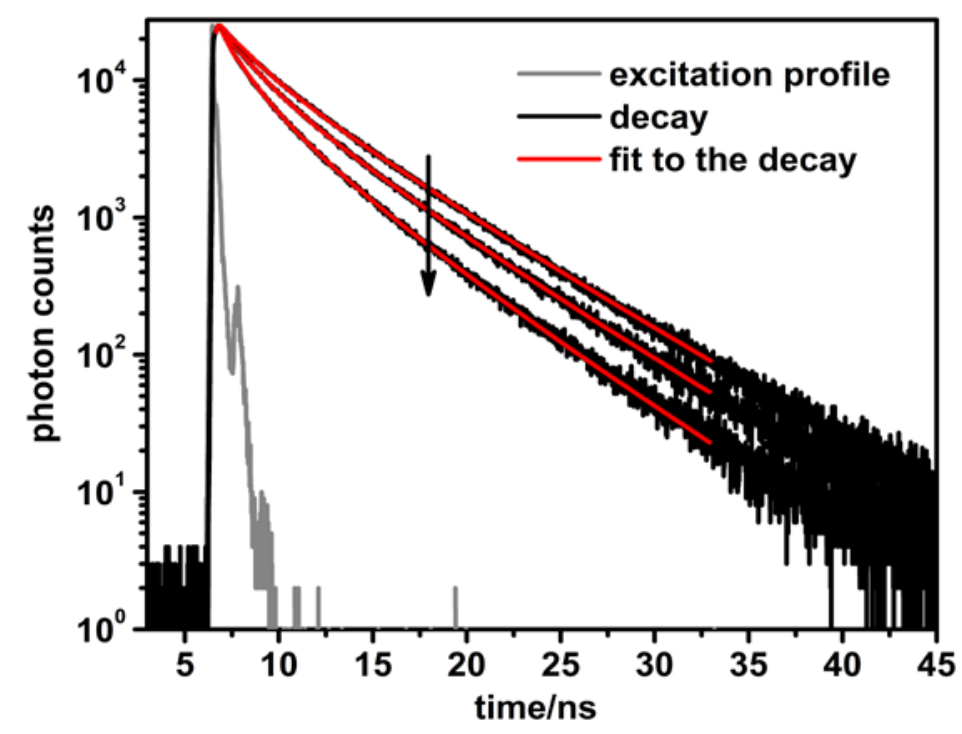

Figure S3: Time-resolved fluorescence decays (black), the fits to these decays (red) and the laser excitation profile (grey). The decays were recorded from g-GM1 donors dissolved in DOPC vesicles at the probe to lipid ratio 1:1000. The $\mathrm{DiD}$ acceptros were mixed at the follwing probe to lipid ratios: 1:400, 1:300 and 1:200 (listed in the order indicated by the arrow, i.e. top to bottom). 